\title{
AUTHOR INDEX VOLUME 28 (2016)
}

Abbasi, A., see Abbasi, S.

Abbasi, A., see Goshvarpour, A.

Abbasi, A., see Goshvarpour, A.

Abbasi, A., see Goshvarpour, A.

Abbasi, S., Abbasi, A. and Sarbaz, Y., Number of Spikes: A Proper Metric for Parallel Fiber

Patterns Recognition by a Purkinje Cell

Ahmad, W. A. W., see Doufesh, H.

Almasganj, F., see Farokhzadi, M.

Al-Zaben, A., A Manometry Catheter Based on Fiber Bragg Gratings: Temperature

Compensation Perspectives and In Vitro Study

Andani, M. E., see Rouhollahi, K.

Ashrafzadehi, F., see Bajestani, G. S.

Azorín, J. C., see Sosa, M.

Badway, S. M., Fkirin, M. A. and Barakat, E. H., Multimodality Comparative Study:

Quantitative Assessment of MRI Contrast Agent Dose Reduction

Bahar, H. B., see Salehpour, P.

Bajestani, G. S., Sheikhani, A., Golpayegani, M. R. H., Ashrafzadehi, F. and Hebrani, P., Cybernetic Approach in Identification of Brain Pattern Variations in Autism Spectrum

Disorder

Barakat, E. H., see Badway, S. M.

Bastami, F., see Salehi, M.

Bernal-Alvarado, J., see Sosa, M.

Bhargavi, V. R. and Senapati, R. K., Curvelet Fusion Enhacement Based Evaluation of

Diabetic Retinopathy by the Identification of Exudates in Optic Color Fundus Images

Bodaghi, T., Karami, M. R. and Jazayeriy, H., VIWA: Computer Interface Device for

Paralyzed People Using Breath Pressure

Breschi, K., see Henriet, J.

Cerrolaza, M., see Gamero, V.

Chang, C.-J., Chern, J.-S., Yang, T.-F. and Yang, S.-W., Age-Related Changes in Postural

Re-Balanced Ability During a Continuous and Unexpected Perturbation Integrated with

Virtual Reality Scenes

Chaudhary, H., see Nayak, C.

Chaudhary, H., see Pathak, V. K.

Chaudhuri, A. and Jayanthi, T., Diagnosis of Cardiac Abnormality Using Heart Sound

Cheikh, R. B., see Trimeche, M.

Chen, P.-C., Chiou, Y.-R., Chen, Y.-F. and Jao, J.-C., Quantifying Active Contour Model

(ACM) Segmented Region Using Edge-Linking and Region-Filling Algorithm (ERA)

Chen, R.-S., see Chu-Su, Y.

Chen, S.-H., see $\mathrm{Yu}, \mathrm{C}$.

Chen, Y.-F., see Chen, P.-C.

Cheng, C.-H., see Lin, Y.-C.

Cheng, H.-Y. K., see Lin, Y.-C.
5 (2016) 1650033

2 (2016) 1650015

4 (2016) 1650024

6 (2016) 1650040

5 (2016) 1650033

6 (2016) 1650043

4 (2016) 1650030

2 (2016) 1650009

4 (2016) 1650026

1 (2016) 1650006

3 (2016) 1650019

1 (2016) 1650001

4 (2016) 1650029

1 (2016) 1650006

1 (2016) 1650001

5 (2016) 1650035

3 (2016) 1650019

6 (2016) 1650046

1 (2016) 1650004

4 (2016) 1650028

3 (2016) 1650017

3 (2016) 1650020

3 (2016) 1650022

5 (2016) 1650037

5 (2016) 1650032

5 (2016) 1650031

4 (2016) 1650023

3 (2016) 1650016

2 (2016) 1650012

4 (2016) 1650023

1 (2016) 1650007

1 (2016) 1650007 
Chern, J.-S., see Chang, C.-J.

3 (2016) 1650020

Chiou, Y.-R., see Chen, P.-C.

4 (2016) 1650023

Chu-Su, Y., Liu, C.-S., Chen, R.-S. and Lin, C.-W., Artificial Neural Networks for

Estimating Glomerular Filtration Rate by Urinary Dipstick for Type 2 Diabetic Patients

Córdova, T., see Sosa, M.

Cuevas, A. C., see Sosa, M.

Daneshvar, S., see Goshvarpour, A.

Davila, E., see Gamero, V.

Degbedzui, D. K., Mills, G. A. and Kaufmann, E. E., Development of Uterine Contraction Monitoring System for Resource-Limited Settings

Dehghan, M. M., see Yazdi, A. A.

Doufesh, H., Ibrahim, F., Ismail, N. A. and Ahmad, W. A. W., Adaptive Neuro-Fuzzy Inference System for Predicting Alpha Band Power of EEG During Muslim Prayer (Salat)

Ebrahimnezhad, H., see Salehpour, P.

Ebrahimzadeh, E., see Nikravan, M.

Elhefnawi, M., see Sheha, M. A.

Esteki, A., see Yazdi, A. A.

Fallah, A., see Farokhzadi, M.

Fang, J.-T., see $\mathrm{Wu}$, J.-H.

Farokhzadi, M., Maleki, A., Fallah, A., Almasganj, F. and Rashidi, S., An Efficient

Hierarchical Structure for Recognition of the Reaching Movements in 3D Space

Farzamfar, S., see Salehi, M.

Firoozan, M. S., Niroomand-Oscuii, H. and Tajabadi, M., Hemodynamic and Structural

Study of Various Bioprosthetic Aortic Heart Valve

Fkirin, M. A., see Badway, S. M.

Foroughipour, M., see Yazdi, M. B.

Gamero, V., Valdivia, J., Davila, E. and Cerrolaza, M., Influence of the Geometry of the Attaching Pegs of the Femoral Component of a Knee Prosthesis

Gautham, A. and Karthik Raj, V., Designing of a Single Arm Single Lead ECG System for

Wet and Dry Electrode: A Comparison with Traditional System

Ghomsheh, F. T., see Yazdi, A. A.

Gogna, A., see Li, C.

Golpayegani, M. R. H., see Bajestani, G. S.

Gong, L., Yang, R., Zhang, C., Liu, Q., Lee, H. and Yang, G., Re-Ranking for Prioritization of Disease-Related Genes

Goshvarpour, A., Abbasi, A. and Goshvarpour, A., Dynamical Analysis of Emotional States from Electroencephalogram Signals

Goshvarpour, A., Abbasi, A. and Goshvarpour, A., Gender Differences in Response to Affective Audio and Visual Inductions: Examination of Nonlinear Dynamics of Autonomic Signals

Goshvarpour, A., Abbasi, A., Goshvarpour, A. and Daneshvar, S., A Novel Signal-Based

Fusion Approach for Accurate Music Emotion Recognition

Goshvarpour, A., see Goshvarpour, A.

Goshvarpour, A., see Goshvarpour, A.

Goshvarpour, A., see Goshvarpour, A.

Hadjer, Z., see Slimane, S. O.

Haghpanahi, M., see Razaghi, R.

Hahn, M. E., see Nakamura, B. H.

Hahn, M. E., see Resseguie, S. C.

Hebrani, P., see Bajestani, G. S.

3 (2016) 1650016

3 (2016) 1650019

3 (2016) 1650019

6 (2016) 1650040

3 (2016) 1650017

6 (2016) 1650045

3 (2016) 1650018

6 (2016) 1650043

4 (2016) 1650029

6 (2016) 1650042

6 (2016) 1650044

3 (2016) 1650018

4 (2016) 1650030

5 (2016) 1650036

4 (2016) 1650030

5 (2016) 1650035

1 (2016) 1650008

1 (2016) 1650001

4 (2016) 1650025

3 (2016) 1650017

3 (2016) 1650021

3 (2016) 1650018

1 (2016) 1650003

1 (2016) 1650006

4 (2016) 1650027

2 (2016) 1650015

4 (2016) 1650024

6 (2016) 1650040

2 (2016) 1650015

4 (2016) 1650024

6 (2016) 1650040

2 (2016) 1650010

2 (2016) 1650014

6 (2016) 1650041

2 (2016) 1650011

1 (2016) 1650006 
Henriet, J., Lang, C., Muthada Pottayya, R. and Breschi, K., A Self-Adaptable Distributed CBR Version of the Equivox System

Huang, P.-S., see Wu, J.-H.

Huang, Y.-C., Lin, P.-W., Qiu, W.-J. and Yang, T.-I, Amphiphilic Polymer-Assisted Synthesis of Hydroxyapatite Particles and Their Influence on the Rheological and Mechanical Properties of Thermosensitive Hydrogels

Ibrahim, F., see Doufesh, H.

Ismail, N. A., see Doufesh, H.

Izadi, M. R., see Nikravan, M.

Jao, J.-C., see Chen, P.-C.

Jayanthi, T., see Chaudhuri, A.

Jazayeriy, H., see Bodaghi, T.

Jian, Y.-J., see Wu, J.-H.

Jin, L., see Resseguie, S. C.

Karami, M. R., see Bodaghi, T.

Karami, V., Mahdavifar (Khayati), R. and Nabavi, S. M., Association Assessment Between Diffusion Tensor Magnetic Resonance Imaging Indices and Clinical Disabilities in MS Patients

Karami, V., see Tadayon, E.

Karbassi, S. M., see Rouhollahi, K.

Karimian, G., see Salehpour, P.

Karthik Raj, V., see Gautham, A.

Kaufmann, E. E., see Degbedzui, D. K.

Keskes, H., see Trimeche, M.

Khalilzadeh, M. M., see Yazdi, M. B.

Khazaei, D., Setarehdan, S. K. and Mehran, Y. Z., The Effectiveness of Music on Human Biological Signals

Lang, C., see Henriet, J.

Lee, H., see Gong, L.

Li, C., Teo, J. Y., Wu, J., Gogna, A., Tan, B. S., Ooi, L. L., Liu, J. and Yu, H., Creation of Clinically-Differential Tumor Mimic Model Using Vaseline-Based Materials with Barium Sulfate for the Validation of Real-Time Ultrasound Image-Guided Liver Biopsy System

Lin, C.-W., see Chu-Su, Y.

Lin, P.-W., see Huang, Y.-C.

Lin, Y.-C., Tsai, L.-C., Cheng, H.-Y. K., Lin, Y.-H. and Cheng, C.-H., Postural Steadiness After Prolonged Standing on Different Sloped Surface in Young Healthy Adults

Lin, Y.-H., see Lin, Y.-C.

Liu, C.-S., see Chu-Su, Y.

Liu, J., see Li, C.

Liu, Q., see Gong, L.

Lo, P.-C. and Tian, W. J. M., Neurocardiac-Cardiorespiratory Interaction of Heart-Brain Mailuns Synchrony at Deep Zen Meditation

Mabrouk, M. S., see Sheha, M. A.

Mahdavifar (Khayati), R., see Karami, V.

Mahdavifar (Khayati), R., see Tadayon, E.

Maleki, A., see Farokhzadi, M.

Mallakzadeh, M., see Razaghi, R.

Mehran, Y. Z., see Khazaei, D.

Mekaoui, S., see Slimane, S. O.

Mesa, F., see Sosa, M.

Mikaeili, M., see Nikravan, M.
4 (2016) 1650028

5 (2016) 1650036

2 (2016) 1650013

6 (2016) 1650043

6 (2016) 1650043

6 (2016) 1650042

4 (2016) 1650023

5 (2016) 1650032

1 (2016) 1650004

5 (2016) 1650036

2 (2016) 1650011

1 (2016) 1650004

5 (2016) 1650034

5 (2016) 1650038

4 (2016) 1650026

4 (2016) 1650029

3 (2016) 1650021

6 (2016) 1650045

5 (2016) 1650031

4 (2016) 1650025

1 (2016) 1650002

4 (2016) 1650028

4 (2016) 1650027

1 (2016) 1650003

3 (2016) 1650016

2 (2016) 1650013

1 (2016) 1650007

1 (2016) 1650007

3 (2016) 1650016

1 (2016) 1650003

4 (2016) 1650027

6 (2016) 1650039

6 (2016) 1650044

5 (2016) 1650034

5 (2016) 1650038

4 (2016) 1650030

2 (2016) 1650014

1 (2016) 1650002

2 (2016) 1650010

3 (2016) 1650019

6 (2016) 1650042 
Mills, G. A., see Degbedzui, D. K.

6 (2016) 1650045

Mojiri, M., see Rouhollahi, K.

4 (2016) 1650026

Muthada Pottayya, R., see Henriet, J.

4 (2016) 1650028

Nabavi, S. M., see Karami, V.

Nabavi, S. M., see Tadayon, E.

Nakamura, B. H. and Hahn, M. E., Myoelectric Activation Differences in Transfemoral

Amputees During Locomotor State Transitions

5 (2016) 1650034

5 (2016) 1650038

6 (2016) 1650041

5 (2016) 1650037

Nayak, C., Singh, A., Chaudhary, H. and Tripathi, A., A Novel Approach for Customized Prosthetic Socket Design

Nikravan, M., Ebrahimzadeh, E., Izadi, M. R. and Mikaeili, M., Toward a Computer Aided Diagnosis System for Lumbar Disc Herniation Disease Based on MR Images Analysis

Niroomand-Oscuii, H., see Firoozan, M. S.

Ooi, L. L., see Li, C.

Oudadess, H., see Trimeche, M.

Pathak, V. K., Nayak, C., Singh, A. K. and Chaudhary, H., A Virtual Reverse Engineering Methodology for Accuracy Control of Transtibial Prosthetic Socket

Qiu, W.-J., see Huang, Y.-C.

Rashidi, S., see Farokhzadi, M.

Razaghi, R., Mallakzadeh, M. and Haghpanahi, M., Dynamic Simulation and Finite Element Analysis of the Maxillary Bone Injury Around Dental Implant During Chewing Different Food

Rebaï, T., see Trimeche, M.

Resseguie, S. C., Jin, L. and Hahn, M. E., Analysis of Dynamic Balance Control in Transtibial Amputees with Use of a Powered Prosthetic Foot

Rouhollahi, K., Andani, M. E., Karbassi, S. M. and Mojiri, M., Design of Robust State Feedback Controllers for Rehabilitation in Parkinsons Tremor: A Simulation Study with Uncertain Model of Basal Ganglia

Salehi, M., Farzamfar, S., Bastami, F. and Tajerian, R., Fabrication and Characterization of Electrospun PLLA/Collagen Nanofibrous Scaffold Coated with Chitosan to Sustain Release of Aloe Vera Gel for Skin Tissue Engineering

Salehpour, P., Bahar, H. B., Karimian, G. and Ebrahimnezhad, H., Adapted Bit-Plane Probability and Wavelet-Based Ulcer Detection in Wireless Capsule Endoscopy Images

Sarbaz, Y., see Abbasi, S.

Senapati, R. K., see Bhargavi, V. R.

Setarehdan, S. K., see Khazaei, D.

Sheha, M. A., Mabrouk, M. S. and Elhefnawi, M., Detecting and Analyzing Copy Number Alternations in Array-Based CGH Data

Sheikhani, A., see Bajestani, G. S.

Shimawaki, S. and Yusa, K., Effect of Measurement Position on Cardio-Ankle Vascular Index (CAVI)

Singh, A., see Nayak, C.

Singh, A. K., see Pathak, V. K.

Slimane, S. O., Talha, M. K., Hadjer, Z. and Mekaoui, S., Comparison of Time and

Frequency Methods for Cardiac Anomalies Recognition

Smaoui, H., see Trimeche, M.

Smida, M., see Trimeche, M.

Sokouti, B., see Sokouti, M.

Sokouti, M. and Sokouti, B., Artificial Intelligent Systems Application in Cervical Cancer Pathological Cell Image Classification Systems: A Review

3 (2016) 1650022

6 (2016) 1650042

1 (2016) 1650008

1 (2016) 1650003

5 (2016) 1650031

5 (2016) 1650037

2 (2016) 1650013

4 (2016) 1650030

2 (2016) 1650014

5 (2016) 1650031

2 (2016) 1650011

4 (2016) 1650026

5 (2016) 1650035

4 (2016) 1650029

5 (2016) 1650033

6 (2016) 1650046

1 (2016) 1650002

6 (2016) 1650044

1 (2016) 1650006

1 (2016) 1650005

3 (2016) 1650022

5 (2016) 1650037

2 (2016) 1650010

5 (2016) 1650031

5 (2016) 1650031

2 (2016) 1630001

2 (2016) 1630001 
Sosa, M., Bernal-Alvarado, J., Azorín, J. C., Cuevas, A. C., Córdova, T. and Mesa, F., Experimental and Mathematical Modeling of Half-Value Layer Measurements for X-Ray Equipment

Tadayon, E., Mahdavifar (Khayati), R., Karami, V. and Nabavi, S. M., A Novel Method for Automatic Classification of Multiple Sclerosis Lesion Subtypes Using Diffusion Tensor MR Images

Tajabadi, M., see Firoozan, M. S.

Tajerian, R., see Salehi, M.

Talha, M. K., see Slimane, S. O.

Tan, B. S., see Li, C.

Teo, J. Y., see Li, C.

Tian, W. J. M., see Lo, P.-C.

Trimeche, M., Smaoui, H., Cheikh, R. B., Smida, M., Rebaï, T., Keskes, H. and Oudadess, H., Elaboration and Evaluation of a Composite Bone Substitute Based on $\beta$-TCP/DCPD and PHBV, Preliminary Results

Tripathi, A., see Nayak, C.

Tsai, L.-C., see Lin, Y.-C.

Urisu, T., Incubation Type Planar Patch Clamp as a New Potential Technology for Developing Neuronal Network High Throughput Screening Devices

Valdivia, J., see Gamero, V.

Wang, G., see $\mathrm{Yu}, \mathrm{C}$.

Wang, Y., see $\mathrm{Yu}, \mathrm{C}$.

$\mathrm{Wu}$, J., see Li, C.

Wu, J.-H., Huang, P.-S., Jian, Y.-J. and Fang, J.-T., Gender Classification by Using Human Nose Features

Yang, G., see Gong, L.

Yang, R., see Gong, L.

Yang, S.-W., see Chang, C.-J.

Yang, T.-F., see Chang, C.-J.

Yang, T.-I, see Huang, Y.-C.

Yazdi, A. A., Esteki, A., Dehghan, M. M. and Ghomsheh, F. T., The Effect of Displacement Rate on Viscoelastic Properties of Rat Cervix

Yazdi, M. B., Khalilzadeh, M. M. and Foroughipour, M., MRI Segmentation by Fuzzy Clustering Method Based on Prior Knowledge

Yu, C., Chen, S.-H., Wang, G. and Wang, Y., Numerical Analysis of Flow Characteristics for the Normal Human Upper Airway

$\mathrm{Yu}, \mathrm{H}$. , see Li, C.

Yusa, K., see Shimawaki, S.

Zhang, C., see Gong, L.
3 (2016) 1650019

5 (2016) 1650038

1 (2016) 1650008

5 (2016) 1650035

2 (2016) 1650010

1 (2016) 1650003

1 (2016) 1650003

6 (2016) 1650039

5 (2016) 1650031

3 (2016) 1650022

1 (2016) 1650007

3 (2016) 1630002

3 (2016) 1650017

2 (2016) 1650012

2 (2016) 1650012

1 (2016) 1650003

5 (2016) 1650036

4 (2016) 1650027

4 (2016) 1650027

3 (2016) 1650020

3 (2016) 1650020

2 (2016) 1650013

3 (2016) 1650018

4 (2016) 1650025

2 (2016) 1650012

1 (2016) 1650003

1 (2016) 1650005

4 (2016) 1650027 